\title{
Scanning SQUID microscopy of vortex clusters in multiband superconductors
}

\author{
Taichiro Nishio, ${ }^{1}$ Vu Hung Dao, ${ }^{1}$ Qinghua Chen, ${ }^{1}$ Liviu F. Chibotaru, ${ }^{1}$ Kazuo Kadowaki, ${ }^{2}$ and Victor V. Moshchalkov ${ }^{1, *}$ \\ ${ }^{1}$ Institute for Nanoscale Physics and Chemistry (INPAC), Katholieke Universiteit Leuven, Celestijnenlaan 200D, \\ B-3001 Leuven, Belgium \\ ${ }^{2}$ Institute of Materials Science, University of Tsukuba, Tsukuba, Ibaraki 305-8573, Japan
}

(Received 22 October 2009; published 19 January 2010)

\begin{abstract}
In type-1.5 superconductors, vortices emerge in clusters, which grow in size with increasing magnetic field. These vortex clusters and their field dependence are directly visualized by scanning superconducting quantum interference device microscopy at very low vortex densities in $\mathrm{MgB}_{2}$ single crystals. Our observations are elucidated by simulations based on a two-gap Ginzburg-Landau theory in the type-1.5 regime.
\end{abstract}

DOI: 10.1103/PhysRevB.81.020506

PACS number(s): 74.25.Uv, 74.70.Ad

In magnesium diboride, ${ }^{1} \mathrm{MgB}_{2}$, the superconducting gaps open for both the two-dimensional (2D) $\sigma$ band [gap size $\Delta_{\sigma}=7.1 \mathrm{meV}$ (Refs. 2 and 3)] and the three-dimensional $\pi$ band $\left[\Delta_{\pi}=2.2 \mathrm{meV}\right.$ (Refs. 3 and 4 )]. The possibility of type-1.5 superconductivity ${ }^{5}$ has been suggested for clean single crystals of $\mathrm{MgB}_{2}$, which lie in the type-1.5 regime: $\kappa_{\sigma}=3.7>1 / \sqrt{2} \quad\left(\right.$ type-2) and $\kappa_{\pi}=0.66<1 / \sqrt{2} \quad$ (type- 1$)$, where $\kappa_{\alpha}(\alpha=\sigma, \pi)$ is the Ginzburg-Landau (GL) parameter for each band estimated from the band-structure calculations. $^{6}$ In contrast to conventional type-2 superconductors, ${ }^{7}$ in type-1.5 superconductors, vortex stripes and gossamerlike vortex patterns, i.e., vortex clusters emerge at relatively low applied fields, ${ }^{5}$ which is due to a competition between attractive (type-1) and repulsive (type-2) vortex interactions governed by a two-gap GL theory. ${ }^{8-12}$ Interestingly, a substantial difference in vortex structure between type-1.5 and type- 2 superconductors, as a fingerprint of type1.5 superconductivity, is expected at very low vortex densities because the intervortex distances in clusters are likely to be almost independent of the applied field in type-1.5 superconductors, whereas the intervortex distances in type-2 superconductors follow the conventional dependence $\left(\phi_{0} / B\right)^{1 / 2}$, where $\phi_{0}\left(=2.07 \times 10^{-15} \mathrm{~T} \mathrm{~m}\right)$ is the flux quantum and $B$ the magnetic field. This motivates strongly direct vortex visualization experiments aimed at investigating vortex structure at very low vortex densities in $\mathrm{MgB}_{2}$ single crystals. Additionally, it is important to verify the existence of type- 1.5 superconductivity in high quality $\mathrm{MgB}_{2}$ crystals different from the ones used in Ref. 5.

In order to study vortex structure in $\mathrm{MgB}_{2}$, we made scans with a scanning superconducting quantum interference device (SQUID) microscope on single crystals of $\mathrm{MgB}_{2}$ which were grown by a pressure synthesis technique ${ }^{13}$ (a crystal grown by this technique was used elsewhere ${ }^{14}$ ). The superconducting transition temperatures $T_{c}$ of the crystals measured by a SQUID magnetometer are $38.5 \mathrm{~K}$ [the transition width $\Delta T_{c}=0.8 \mathrm{~K}(10-90 \%$ criterion $\left.)\right]$. The surface of crystals was confirmed not to have any cracks or holes by using a field-emission scanning electron microscope in the secondary electron regime. High-resolution transmission electron microscope images and electron-diffraction patterns for the crystals show that the crystals have no grain boundaries. No impurity contamination was detected within $0.1 \%$ accuracy by an electron probe microanalyzer.
Our scanning SQUID microscope is based on prototype SQM2000 (Ref. 15) (SII NanoTechnology Inc.). We fabricated a SQUID sensor, which is a SQUID magnetometer linked with a circular $\mathrm{Nb}$ pickup loop. The $\mathrm{Nb}$ pickup loop with an inner diameter of $8 \mu \mathrm{m}$ scans the surface of a sample, keeping a few micrometers away from the surface. A magnetic-flux sensitivity is $5 \mu \phi_{0} / \mathrm{Hz}^{1 / 2}$. A field noise referred to the pickup loop is $\sim 10^{-10} \mathrm{~T} / \mathrm{Hz}^{1 / 2}$. A spatial resolution is $\sim 2.5 \mu \mathrm{m}$. Before scans, as-grown crystals were cooled from a temperature higher than $T_{c}$ to $4.2 \mathrm{~K}$ under the magnetic field parallel to the $c$-axis and then scans were made on the $a b$-plane at $4.2 \mathrm{~K}$. Scanning steps are 0.5 and $1.0 \mu \mathrm{m}$ for $\mathrm{MgB}_{2}$ single crystals and a $\mathrm{Nb}$ film, respectively.

We model vortex systems in a type- 1.5 superconductor in the same way as in Ref. 5. We invoke the following two-gap GL theory to derive the vortex-vortex interaction numerically. A two-gap GL free-energy functional ${ }^{8-12}$ is the sum of two single-band GL functionals with the Josephson coupling term corresponding to the interaction between two bands,

$$
F=\int d r^{3}\left(F_{\sigma}+F_{\pi}+F_{\sigma \pi}+\frac{h^{2}}{2 \mu_{0}}\right),
$$

where $F_{\alpha}(\alpha=\sigma, \pi)$ is the free energy of each band and $h(=|\mathbf{h}|)$ is a magnetic field $(\mathbf{h}=\operatorname{rot} \mathbf{A}$, where $\mathbf{A}$ is the vector potential), ${ }^{16}$

$$
F_{\alpha}=2 E_{\alpha}\left|\Psi_{\alpha}\right|^{2}+\left|E_{\alpha}\right|\left|\Psi_{\alpha}\right|^{4}+C\left|\left(-i \nabla+\frac{2 \pi}{\phi_{0}} \mathbf{A}\right) \Psi_{\alpha}\right|^{2},
$$

where $E_{\alpha}$ is the condensation energy ${ }^{17}$ and $C=\left(\phi_{0} / 2 \pi\right) \sqrt{\left|E_{\alpha}\right| / \mu_{0} \kappa_{\alpha}^{3}} . F_{\sigma \pi}$ is the Josephson coupling term: $F_{\sigma \pi}=\frac{E_{\gamma}}{2}\left(\Psi_{\sigma}^{*} \Psi_{\pi}+\Psi_{\pi}^{*} \Psi_{\sigma}\right)$, where $E_{\gamma}$ is the coupling energy, where we take a value used in Ref. 17. The vortex-vortex interaction energy between two vortices is the sum of increased energies of each vortex caused by the presence of the other. It can be derived by numerically minimizing the twogap GL free energy of two vortices with a variational procedure. ${ }^{18}$ The minimization gives the interaction consisting of short-range repulsion and weak long-range attraction, which is similar to that reported in Ref. 18.

An equation of motion for a vortex $i$ is $\mathbf{F}_{i}=\mathbf{F}_{i}^{v v}+\mathbf{F}_{i}^{T}=\eta \mathbf{v}_{i}$, where $\mathbf{F}_{i}^{v v}$ is the vortex-vortex interaction term we calculate 


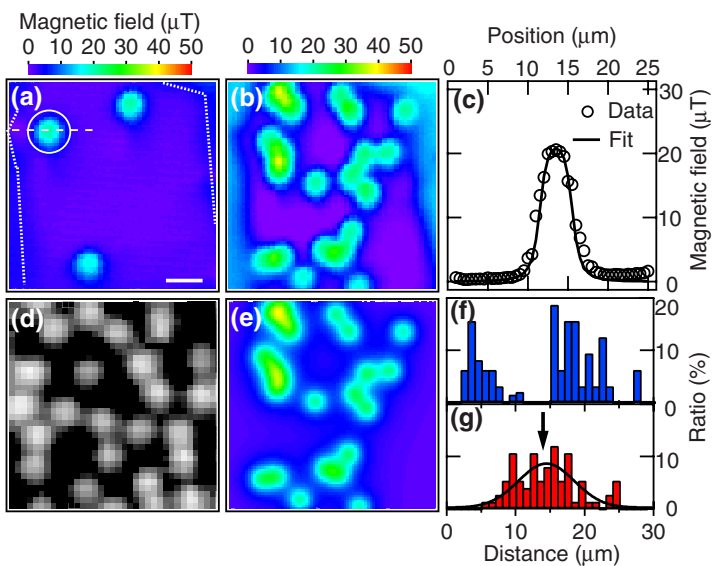

FIG. 1. (Color online) Scanning SQUID microscope images of vortices at (a) $1 \mu \mathrm{T}$ and (b) $10 \mu \mathrm{T}$ for a $\mathrm{MgB}_{2}$ single crystal, the dimensions of which are approximately $80 \times 60 \mu \mathrm{m}^{2}$. Dotted lines show the edges of the sample. A scale bar corresponds to $10 \mu \mathrm{m}$. The integral of the magnetic field within a solid line in (a) gives the flux $\sim \phi_{0}$. (c) The cross section of the vortex image along a broken line in (a) (open circles) and the model fit (solid line) (see text). (d) Scanning SQUID microscope image of vortices at $10 \mu \mathrm{T}$ in a $\mathrm{Nb}$ film with a thickness of $200 \mathrm{~nm}\left(T_{c}=9.1 \mathrm{~K}\right)$. (e) The $2 \mathrm{D}$ multifits of the SQUID image in (b) to numerical calculations by a London model (see text). (f) and (g) The distribution of the intervortex distance derived from (e) and (d), respectively. A solid line in (g) represents a fit to the Gaussian function. An arrow indicates the intervortex distance calculated from $\left(\phi_{0} / B\right)^{1 / 2}$.

above, $\mathbf{F}_{i}^{T}$ the thermal stochastic force which satisfies $\left\langle\mathbf{F}_{i}^{T}(t)\right\rangle=0$ and $\left\langle\mathbf{F}_{i}^{T}(t) \mathbf{F}_{j}^{T}\left(t^{\prime}\right)\right\rangle=2 \eta \delta_{i j} \delta\left(t-t^{\prime}\right) k_{B} T$, and $\eta$ the viscosity, where we take a value estimated in Ref. 5 . The systems with no pinning centers were initially prepared in a high-temperature molten state and then annealed with two million temperature steps. ${ }^{19}$ We made the system stable during 2000 time steps in each step of temperature.

Figures 1(a) and 1(b) show scanning SQUID microscope images of vortices at very low vortex densities for $\mathrm{a} \mathrm{MgB}_{2}$ single crystal. The size of a vortex in the image does not correspond straightforwardly to the penetration depth $\lambda$ but rather to a combination of $\lambda$ and the extent of a stray field emanating from a vortex at the sample surface. In order to estimate $\lambda$ it is necessary to compare the cross section of the vortex image with appropriate numerical calculations where the extent of the stray field is taken into account. ${ }^{15}$ We calculate numerically the spatial distribution of a magnetic field from a vortex above the sample surface, using a London model. ${ }^{15,20,21}$

Figure 1(c) shows a fit of the cross section of a vortex image in Fig. 1(a) by the numerical simulations. We obtain $\lambda=0.01-0.13 \mu \mathrm{m}(z=1.7 \pm 0.1 \mu \mathrm{m}$, where $z$ is the distance between the sample surface and the pickup loop) from the fit. The $z$ value was determined by the fit of a vortex image in a $\mathrm{Nb}$ film (known parameter $\lambda=50 \mathrm{~nm}$ ) mounted on a sample holder with a $\mathrm{MgB}_{2}$ crystal. The range of $\lambda$ is a residual based on the error bar in the $z$ value which was assigned by doubling of the $\chi$ value in the fit in a $\mathrm{Nb}$ film (a doubling of the $\chi$ value only gives statistical errors). Due to the error it is difficult to specify how close our $\lambda$ value is to the band- structure calculations. ${ }^{6}$ However, first-principles calculations $^{6,22}$ of the penetration depth in the clean limit fall on our $\lambda$ value within the error bar.

In type-2 superconductors with the weak pinning, the Abrikosov lattice is often seen in field cooling experiments even if a vortex density is quite low: ${ }^{15}$ the vortex lattice made of vortices strongly interacting with each other at temperatures very close to $T_{c}$ is frozen with decreasing temperature. Vortex patterns at very low vortex densities in a $\mathrm{MgB}_{2}$ single crystal, which has the weak pinning, ${ }^{17}$ seem to be different from that in the conventional weak pinning system. At $B=1 \mu \mathrm{T}$ vortices are located far away from each other.[Fig. 1(a)] However, with increasing applied field vortices come closer to each other to form compact groups as shown in Figs. 1(b) and 1(e), which is never seen in superconductors with the weak pinning, such as $\mathrm{MoGe}$ films ${ }^{15}$ and $\mathrm{NbSe}_{2}$ single crystals. ${ }^{5}$

In a $\mathrm{Nb}$ film with the strong pinning, disordered vortex patterns are observed at low vortex densities, as shown in Fig. 1(d). We now compare qualitatively these vortex patterns. Figure $1(\mathrm{e})$ shows the $2 \mathrm{D}$ multifits ${ }^{23}$ of the scanning SQUID image in Fig. 1(b) to numerical calculations from a London model. ${ }^{20,21}$ Fits were made by parameterizing the coordinates of each position of vortices with $z$ and $\lambda$ determined above. From the fits it turns out that the number of vortices is 23 , which is indeed consistent with 23 estimated by $S B / \phi_{0}$, where $S$ is the area of a sample. The distribution of the intervortex distance can be derived from the fits, as shown in Fig. 1(f). This distribution is a bimodal and it clearly splits into two: one ranging from $\sim 2 \mu \mathrm{m}$ to $\sim 7 \mu \mathrm{m}$ and another from $\sim 15 \mu \mathrm{m}$ to $\sim 30 \mu \mathrm{m}$. In principle, the former corresponds to the intragroup distribution and the latter corresponds to that concerning the intergroup. On the other hand, the vortex distribution in a $\mathrm{Nb}$ film can be fitted by the Gaussian function, as shown in Fig. 1(g). The center of the peak in the Gaussian function is at $14.5 \mu \mathrm{m}$, indicating that the average intervortex distance is $14.5 \mu \mathrm{m}$. This agrees with the intervortex distance $\left(\phi_{0} / B\right)^{1 / 2}$ of $\sim 14 \mu \mathrm{m}$ estimated by assuming the presence of the Abrikosov lattice. This fact indicates that the vortex pattern in Fig. 1(b) is not a disordered Abrikosov lattice but vortex cluster array. On the other hand, the vortex pattern in Fig. 1(d) does correspond to the strongly disordered Abrikosov lattice.

Figures 2(a)-2(c) show the variation of the vortex structure with applied fields. After vortices form local clusters in Fig. 2(a), some islands of magnetic flux and the semiMeissner regions come out of the grouping with increasing applied field. [Figs. 2(b) and 2(c)] The positions of the vortices can be determined by the 2D multifits [Figs. 2(d)-2(f)] of Figs. 2(a)-2(c), as shown in Figs. 2(g)-2(i). The position maps show that vortices line up locally in the early stage [Fig. 2(g)] and then small vortex clusters merge with each other, [Fig. 2(h)] to grow into bigger clusters with applied fields. [Fig. 2(i)] The number of vortices changes to 49 in Fig. 2(h) and then 114 in Fig. 2(i). These are reasonably well approximated by 46 and 116 estimated from $S B / \phi_{0}$, respectively.

We note that the vortex array varies considerably in the locations of clusters measured by different cool downs, while 


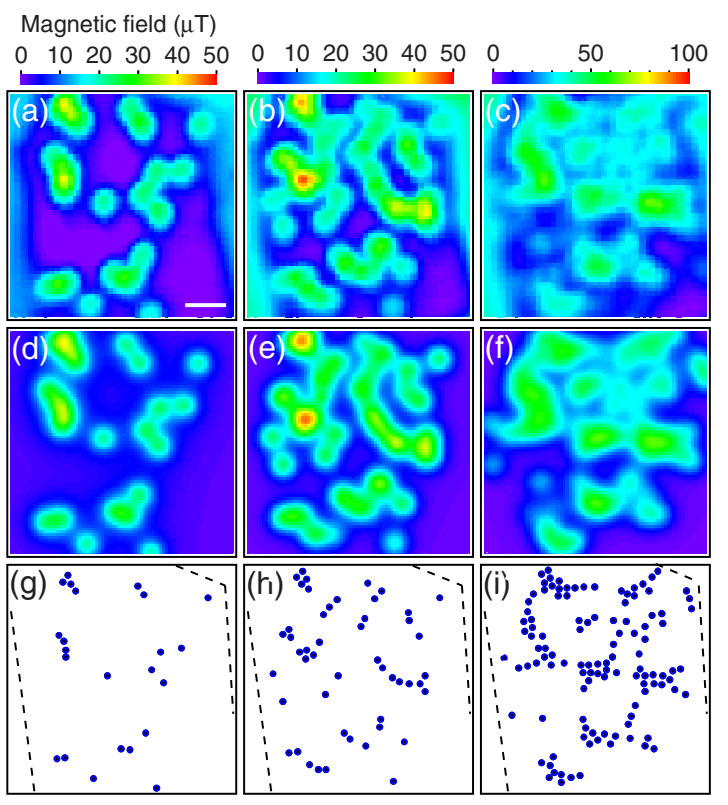

FIG. 2. (Color online) Scanning SQUID microscope images of vortices at (a) $10 \mu \mathrm{T}$, (b) $20 \mu \mathrm{T}$, and (c) $50 \mu \mathrm{T}$ for a $\mathrm{MgB}_{2}$ single crystal. A scale bar corresponds to $10 \mu \mathrm{m}$. (d)-(f) The 2D multifits of the SQUID images in (a)-(c) by a London model (see text). (g)-(i) The locations of vortices in (d)-(f) obtained by the 2D multifits of the images. Broken lines show the edges of the sample.

keeping the same general features, as seen in Fig. 3. Since the pinning sites are fixed, this implies that the vortex clustering is not due to the presence of pinning centers but is rather to be attributed to the peculiar vortex-vortex interaction combining repulsion and attraction.

Figures 4(a)-4(d) show results of the simulation mentioned above. In type- 1.5 superconductors vortices begin to form clusters consisting of a few vortices at very low vortex densities, [Fig. 4(a)] which does not coincide with the experiment displayed in Fig. 1(a). Perhaps, the existence of some pinning centers may be responsible for this fact. This will be considered in the future work. The number of small clusters increases with applied fields, [Fig. 4(b)] which apparently corresponds to Fig. 2(g). Then small clusters start to merge with each other,[Fig. 4(c)] to grow into bigger clusters with a further increase of an applied field [Fig. 4(d)]. This simulation result coincides quite well with experimental data [Figs. 2(h) and 2(i)].
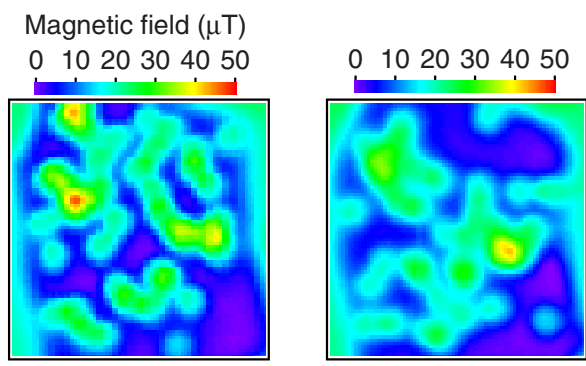

FIG. 3. (Color online) Scanning SQUID microscope images of vortices at $20 \mu \mathrm{T}$ obtained from different cool downs. The left image is the same as Fig. 2(b).

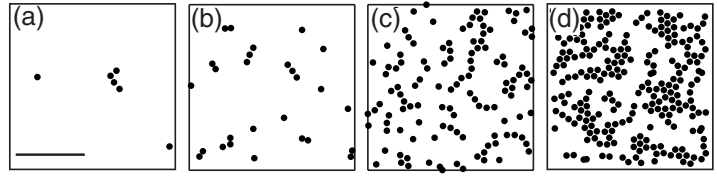

FIG. 4. Simulations of systems consisting of (a) 6, (b) 27, (c) 122 , and (d) 500 vortices which have the interaction energy calculated from a two-gap GL theory for the type-1.5 condition (see text). A scale bar corresponds to $10 \mu \mathrm{m}$.

The simulations show that in a vortex cluster the distances between vortices and their nearest neighbors are almost independent of the applied field $(\sim 1 \mu \mathrm{m})$. Therefore, these distances are not determined by the expression $\left(\phi_{0} / B\right)^{1 / 2}$ but rather by the competition between short-range repulsive and long-range attractive interactions between vortices. In Fig. 5 the average intervortex distance in clusters obtained from scanning SQUID and decoration images ${ }^{5}$ is shown as a function of applied field, compared with the dependence $\left(\phi_{0} / B\right)^{1 / 2}$. The average distances indeed do not follow any dependence close to $\left(\phi_{0} / B\right)^{1 / 2}$ but follow instead a very weak linear field dependence. The intervortex distance in clusters, independent of the applied field, can be identified as a unique property of a system of vortices with short-range repulsion and long-range attraction, i.e., vortices induced in a type-1.5 superconductor. This fact indicates that vortex clusters definitely emerge even in crystals grown by a different crystal maker, implying that in general vortices tend to form vortex clusters in high quality single crystals of $\mathrm{MgB}_{2}{ }^{5}$

A difference between $\left(\phi_{0} / B\right)^{1 / 2}$ and the intervortex distance in clusters decreases as an applied field increase and these two length scales become equivalent around $0.6 \mathrm{mT}$. Any vortex cluster is unlikely to be observed above $0.6 \mathrm{mT}$. In fact Moshchalkov et al. ${ }^{5}$ reported no clearly visible vortex clusters at $1.0 \mathrm{mT}$. The observations of long-range Abrikosov lattices at $20 \mathrm{mT}$ and $0.2 \mathrm{~T}$ (Refs. 4 and 24) are consistent with this scenario. Although Vinnikov et al. ${ }^{24}$ obtained a decoration image of a disordered vortex lattice at $0.44 \mathrm{mT}$, it would be too difficult to find vortex clusters, a size of which is more than $20 \mu \mathrm{m}$ around $0.5 \mathrm{mT}$, as reported in Ref. 5 .

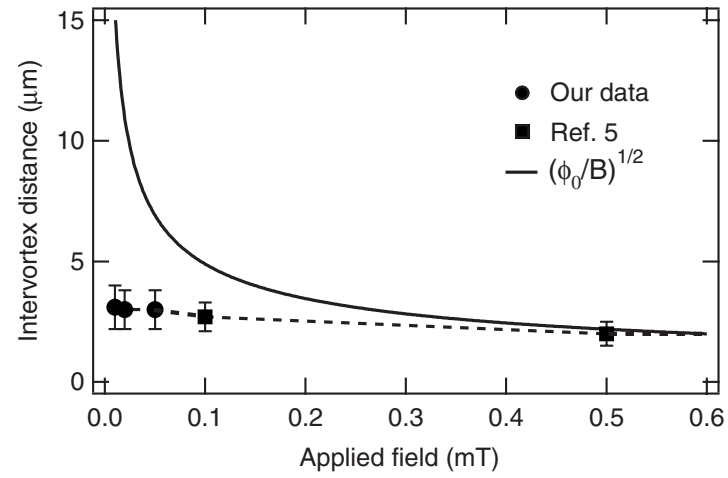

FIG. 5. The average intervortex distance in vortex clusters as a function of applied field. These distances are obtained from fits of scanning SQUID images in Figs. 2(g)-2(i) (filled circles) and decoration images (Ref. 5) at $0.1 \mathrm{mT}$ and $0.5 \mathrm{mT}$ (filled squares). Error bars indicate the range of the distribution of vortex distances in clusters. A solid line represents the dependence $\left(\phi_{0} / B\right)^{1 / 2}$. 
In conclusion, scanning SQUID microscopy shows that in high quality $\mathrm{MgB}_{2}$ single-crystal vortices line up locally at very low vortex densities and grow into vortex clusters with increasing applied field. The average distances between nearest neighboring vortices forming clusters are practically independent of the applied field $B$, in contrast to the conventional $(1 / B)^{1 / 2}$ behavior. The simulations of a vortex system of a type-1.5 superconductor indeed reproduce these observations. Our experimental data, as well as consistency between experiment and the simulations, clearly demonstrates that high quality $\mathrm{MgB}_{2}$ single crystals lie in the type1.5 regime.

This work was supported by Methusalem Funding by the Flemish government, the Flemish FWO, the Belgian Interuniversity Attraction Poles (IAP), the ESF-NES "Nanoscience and Engineering in Superconductivity" Programmes, and JSPS core-to-core NES program.
*Corresponding author; victor.moshchalkov@fys.kuleuven.be

${ }^{1}$ J. Nagamatsu, N. Nakagawa, T. Muranaka, Y. Zenitani, and J. Akimitsu, Nature (London) 410, 63 (2001).

${ }^{2}$ M. Iavarone, G. Karapetrov, A. E. Koshelev, W. K. Kwok, G. W. Crabtree, D. G. Hinks, W. N. Kang, E. M. Choi, H. J. Kim, H. J. Kim, and S. I. Lee, Phys. Rev. Lett. 89, 187002 (2002); P. Szabó, P. Samuely, J. Kačmarčík, T. Klein, J. Marcus, D. Fruchart, S. Miraglia, C. Marcenat, and A. G. M. Jansen, ibid. 87, 137005 (2001).

${ }^{3}$ F. Giubileo, D. Roditchev, W. Sacks, R. Lamy, D. X. Thanh, J. Klein, S. Miraglia, D. Fruchart, J. Marcus, and P. Monod, Phys. Rev. Lett. 87, 177008 (2001).

${ }^{4}$ M. R. Eskildsen, M. Kugler, S. Tanaka, J. Jun, S. M. Kazakov, J. Karpinski, and Ø. Fischer, Phys. Rev. Lett. 89, 187003 (2002); G. Rubio-Bollinger, H. Suderow, and S. Vieira ibid. 86, 5582 (2001).

${ }^{5}$ V. V. Moshchalkov, M. Menghini, T. Nishio, Q. H. Chen, A. V. Silhanek, V. H. Dao, L. F. Chibotaru, N. D. Zhigadlo, and J. Karpinski, Phys. Rev. Lett. 102, 117001 (2009).

${ }^{6}$ I. I. Mazin, O. K. Andersen, O. Jepsen, O. V. Dolgov, J. Kortus, A. A. Golubov, A. B. Kuz'menko, and D. van der Marel, Phys. Rev. Lett. 89, 107002 (2002).

${ }^{7}$ E. H. Brandt, Rep. Prog. Phys. 58, 1465 (1995).

${ }^{8}$ I. N. Askerzade, A. Gencer, and N. Güçlü, Supercond. Sci. Technol. 15, L17 (2002).

${ }^{9}$ E. Babaev, Phys. Rev. Lett. 89, 067001 (2002).

${ }^{10}$ E. Babaev and M. Speight, Phys. Rev. B 72, 180502(R) (2005).
${ }^{11}$ E. Babaev and N. W. Ashcroft, Nat. Phys. 3, 530 (2007).

${ }^{12}$ M. E. Zhitomirsky and V. H. Dao, Phys. Rev. B 69, 054508 (2004).

${ }^{13}$ Y. Machida, S. Sasaki, H. Fujii, M. Furuyama, I. Kakeya, and K. Kadowaki, Phys. Rev. B 67, 094507 (2003).

${ }^{14}$ S. Souma, Y. Machida, T. Sato, T. Takahashi, H. Matsui, S. C. Wang, H. Ding, A. Kaminski, J. C. Campuzano, S. Sasaki, and K. Kadowaki, Nature (London) 423, 65 (2003).

${ }^{15}$ T. Nishio, S. Okayasu, J. I. Suzuki, N. Kokubo, and K. Kadowaki, Phys. Rev. B 77, 052503 (2008).

${ }^{16} \mathrm{M}$. Tinkham, Introduction to Superconductivity, 2nd ed. (McGraw-Hill, New York, 1996).

${ }^{17}$ M. Eisterer, Supercond. Sci. Technol. 20, R47 (2007).

${ }^{18}$ L. Jacobs and C. Rebbi, Phys. Rev. B 19, 4486 (1979).

${ }^{19}$ C. Reichhardt, C. T. Olson, I. Martin, and A. R. Bishop, Europhys. Lett. 61, 221 (2003).

${ }^{20}$ V. G. Kogan, V. V. Dobrovitski, J. R. Clem, Y. Mawatari, and R. G. Mints, Phys. Rev. B 63, 144501 (2001).

${ }^{21}$ J. R. Kirtley, V. G. Kogan, J. R. Clem, and K. A. Moler, Phys. Rev. B 59, 4343 (1999).

${ }^{22}$ A. A. Golubov, A. Brinkman, O. V. Dolgov, J. Kortus, and O. Jepsen, Phys. Rev. B 66, 054524 (2002).

${ }^{23}$ T. Nishio, Q. Chen, W. Gillijns, K. De Keyser, K. Vervaeke, and V. V. Moshchalkov, Phys. Rev. B 77, 012502 (2008).

${ }^{24}$ L. Y. Vinnikov, J. Karpinski, S. M. Kazakov, J. Jun, J. Anderegg, S. L. Bud'ko, and P. C. Canfield, Phys. Rev. B 67, 092512 (2003). 